\title{
Vers la thérapie génique du xeroderma pigmentosum
}

Le xeroderma pigmentosum (XP) est une maladie monogénique à transmission autosomique et récessive où des mutations dans des gènes de réparation de l'ADN provoquent une hypersensibilité du tissu cutané au rayonnement solaire. Le nom de la maladie, venant du latin, signifie littéralement derme sec (xeroderma) pigmenté (pigmentosum). En effet, dès la naissance, la peau des individus XP présente une sécheresse dans la couche cornée et le premier impact avec la lumière provoque des réactions d'hyperpigmentation. Au bout de quelques années, des tumeurs apparaissent dans les parties du corps exposées au soleil, d'abord sous forme bénigne, mais elles dégénèrent en épithéliomas baso- ou spinocellulaires et en mélanomes. Dans les formes les plus graves de la maladie, sont associées à ces affections cutanées des dégénérescences du système nerveux central. L'espérance de vie pour les malades XP les plus sérieusement atteints (environ 25-35\%) ne dépasse pas l'âge de 15 ans [1]. La seule solution thérapeutique de cette maladie a été, jusqu'à présent, la prévention, en mettant l'individu à l'abri de la lumière afin d'éviter la formation de tumeurs, l'ablation chirurgicale des tumeurs et l'utilisation des composés rétinoïdes pour empêcher la progression des cancers cutanés. Une autre possibilité thérapeutique est l'autogreffe d'une partie de la peau du malade non exposée au soleil, en suivant les protocoles classiques de la dermatologie courante. Toutefois, malgré les efforts dans la recherche thérapeutique de cette maladie, aucun progrès n'a été accompli pour un traitement efficace à long terme. Même la greffe de peau non exposée ne peut constituer une thérapie à long terme, car la peau greffée reste génétiquement sensible au soleil et elle développe finalement des cancers.
Depuis les années 1990, le clonage des gènes humains de la réparation de l'ADN a permis l'identification d'au moins 13 gènes impliqués dans la réparation des lésions induites par la lumière solaire, dont 5 impliqués dans la pathologie XP. Parmi ces derniers, au moins $3(X P B, X P D$ et $X P G)$ sont aussi responsables de deux autres maladies de la réparation comme la trichothiodystrophie et le syndrome de Cockayne $[2,3]$. On a montré que certains de ces gènes $(X P B, X P D$ et $T T D-A$, ce dernier, impliqué dans un groupe de complémentation de la trichothiodystrophie, qui n'a pas encore été cloné), faisaient partie de la machinerie transcriptionnelle en agissant avec l'ARN polymérase II, dans le complexe TFIIH, pour régler le début de la transcription [4]. L'identification de ces gènes et des mutations chez les malades a permis de mieux connaître les mécanismes de base de la réparation de l'ADN, notamment, en introduisant la version normale du gène dans les cellules des malades ou en étudiant par les méthodes biochimiques le rôle de chaque protéine de réparation dans des systèmes d'analyse in vitro.

Notre recherche, en collaboration avec le laboratoire de Jean-Michel Heard et Olivier Danos, à l'Institut Pasteur, d'une nouvelle technique de correction génétique des cellules des malades, permet désormais d'évoquer pour la première fois un protocole possible de thérapie génique, à long terme, chez les malades XP. Plusieurs lignées de cellules de malades ont eu une correction génétique à l'aide d'une construction rétrovirale basée sur le virus de Moloney de la leucémie murine (MoMuLV), capable d'intégrer une séquence d'ADN exogène dans les cellules qu'il infecte sans se multiplier dans la cellule hôte (ne présentant donc pas de danger potentiel). Cette correction s'est révélée très efficace, car plusieurs mois après transduction, les cellules des malades, initialement sensibles aux UV, présentent toujours un phénotype normal en ce qui concerne, non seulement leur sensibilité aux UV, mais aussi toutes les autres propriétés cellulaires liées à la réparation de l'ADN. L'analyse de ces fibroblastes diploïdes non transformés mais complémentés pour leur déficit en réparation nous permet de lier directement certains phénotypes retrouvés spécifiquement dans les cellules $\mathrm{XP}$ et la réparation de l'ADN, puisque ces phénotypes sont aussi complémentés par l'expression du gène de réparation normal [5].

Nous avons donc franchi la première étape de thérapie génique cellulaire, en produisant des lignées de fibroblastes de peau de malades au phénotype guéri. Cela constitue la condition sine qua non pour poursuivre les étapes suivantes: (a) la correction génétique des kératinocytes de malades (les cellules souches de l'épiderme); (b) la production in vitro d'un épithélium de culture, génétiquement corrigé qui pourra être le greffon à utiliser pour remplacer la peau exposée chez les malades XP.

M.M.

A.S.

1. Sarasin A, Renault G, Blanchet-Bardon C, Boué J, Dumez Y. Le xeroderma pigmentosum: caractéristiques cliniques génétiques et cellulaires. Développement d'un test anténatal. médecine/sciences 1988; 4: 608-17.

2. Sarasin $\mathrm{A}$. Les gènes humains de la réparation de l'ADN. médecine/sciences 1994; 10: 43-54.

3. Schaeffer L, Egly J. BTF2/TFIIH, un facteur de transcription et réparation impliqué dans des maladies de la réparation de l'ADN. médecine/sciences 1994; $10: 973-8$.

4. Roy R, Bergmann E, Egly J. TFIIH (BTF2), à l'interface de trois processus cellulaires: transcription, réparation et cycle cellulaire. médecine/sciences $1995 ; 11: 879-82$.

5. Carreau M, Quilliet X, Eveno E, Salvetti A, Danos O, Heard JM, Mezzina M, Sarasin A. Functional retroviral vector for gene therapy of xeroderma pigmentosum group D patients. Hum Gene Ther $1995 ; 6$ : 1307-16. 